\title{
Corticosteroids and Insulin Resistance in the ICU
}

\author{
Christopher Pretty,* J. Geoffrey Chase,* Jessica Lin,** Geoffrey Shaw,** Aaron Le Compte,* Normy Razak,* \\ Jacquelyn Parente*
* University of Canterbury, Dept of Mechanical Engineering, Centre for Bioengineering, Christchurch, New Zealand (Tel: +64 3364 2596; e-mail: cgp19@student.canterbury.ac.nz). **University of Otago, Dept of Medicine, Christchurch, New Zealand

\begin{abstract}
Corticosteroids reduce insulin sensitivity in healthy individuals by 30- 62-percent. The aim of this research was to use model-based methods to determine whether this reduction is also true in critically ill patients and how it may affect tight glycaemic control. A clinically validated model-based measure of insulin sensitivity was used to quantify changes between two matched cohorts of 40 intensive care unit (ICU) patients from Christchurch hospital. A 9-percent reduction in median insulin sensitivity was seen between the control cohort and patients receiving corticosteroids (per patient dose equivalent to $160 \mathrm{mg} / \mathrm{d}$ of hydrocortisone). On a per-patient basis 11-22-percent reductions were observed with higher percentile patients having greater suppression of insulin sensitivity. This research has shown that corticosteroids cause a much lower reduction in insulin sensitivity for critically ill patients compared to healthy controls and may thus have far less impact than suspected on glycaemic control in the ICU setting.
\end{abstract}

Keywords: Insulin sensitivity, Physiological models, Glycaemic control, Intensive care, Steroids

\section{INTRODUCTION}

It is known that corticosteroids increase insulin resistance in healthy individuals. However, there is a lack of data about whether this affect is also true for critically ill patients. Insulin resistance, defined by relatively low insulin-mediated glucose disposal, makes tight glycaemic control (TGC) of intensive care unit (ICU) patients more difficult. Treatment with corticosteroids may therefore make this task even harder.

Several studies have reported decreases in the insulin sensitivity of healthy subjects of 30- 62-percent after shortterm administration of dexamethasone (2- or $6 \mathrm{mg} / \mathrm{d}$ ) (Binnert et al. 2004, Larsson \& Ahren 1996, Nicod et al. 2003, Perry et al. 2003). Pagano et al. (1983) document a similar change with prednisone $(15 \mathrm{mg} / \mathrm{d})$. The mechanisms and pathways underlying these dramatic reductions in insulin sensitivity are not yet fully understood. Metabolic adaptations, including enhanced endogenous glucose production (EGP), increased plasma insulin concentrations, and reduced whole-body glucose disposal were also reported in the studies noted above.

Hyperglycaemia is prevalent in critical care (Capes et al. 2000, Chase et al. 2008, McCowen et al. 2001, Mizock 2001, Van den Berghe et al. 2001). Increased secretion of counterregulatory hormones stimulates endogenous glucose production and reduces effective insulin sensitivity (McCowen et al. 2001, Mizock 2001). Tight control of blood glucose has been shown to improve clinical outcomes, reducing the risk of severe infection (Bistrian 2001), myocardial infarction (Capes et al. 2000) and critical illnesses such as multiple organ failure and polyneuropathy (Van den Berghe et al. 2001). More importantly, van den Berghe et al
(2006, 2001), Krinsley (2003) and Chase et al (2008) have shown that tight glucose control can reduce ICU mortality by 18-45-percent.

Corticosteroids are used in critical care to treat a variety of inflammatory and allergic disorders.

\subsection{Hypothesis}

Insulin sensitivity is reduced by corticosteroids in critically ill patients, but potentially to a lesser extent than in healthy individuals.

\section{$1.3 \mathrm{Aim}$}

The aim of this research was to use model-based methods to quantify the affect of corticosteroid therapy on the insulin sensitivity of ICU patients, to determine whether it is detrimental to TGC and patient outcome.

\section{SUBJECTS AND METHODS}

\subsection{Subjects}

A clinically validated, model-based measure of insulin sensitivity $\left(S_{I}\right)$ was used to quantify changes in insulin sensitivity between two matched, critically ill cohorts. This research was conducted as a retrospective study using records from 80 patients admitted to the Christchurch ICU between 2005 and 2007.

A cohort of 40 patients who each spent 24 hours or more on the SPRINT protocol (Chase et al. 2008) and received corticosteroid therapy during this time was selected from the 
available records. These patients were treated with one or more of; hydrocortisone, prednisone, prednisolone, methylprednisolone or dexamethasone, with a median daily dose for the cohort equivalent to $160 \mathrm{mg}$ of hydrocortisone (Derendorf et al. 1993, Melby 1977). These patients were also screened to avoid any use of beta-blockers, as they can affect glucose metabolism (Rizza et al. 1980). Where a patient did not receive steroid therapy for the entire time they were on SPRINT, their insulin sensitivity was considered to be affected by the drug for 12 hours following the last dose, to allow for the plasma concentration to recover.

A matching control cohort that did not receive any corticosteroid or beta-blocker therapy was also selected from patients on the SPRINT protocol. Details of the two study cohorts are shown in Table 1. Importantly, glycaemic control was also matched to avoid any differences this might cause.

The SPRINT protocol is a simple wheel-based system that modulates insulin and nutritional inputs, titrating doses to the patient-specific insulin sensitivity to provide tight glycaemic control (Chase et al. 2008). SPRINT has been used in the Christchurch ICU since August 2005 on more than 1000 patients. The requirement for the patients in this study to be on the SPRINT protocol ensures that they have regular and accurate records of blood glucose levels, insulin administered and nutrition given. The use of these patient records falls under existing ethics approval granted by the Upper South Regional Ethics Committee, New Zealand.

Table 1. Comparison of steroid and control cohorts. Where appropriate, data are shown as median [IQR $\left.{ }^{\dagger}\right]$.

\begin{tabular}{|l|c|c|c|}
\hline & $\begin{array}{c}\text { Control } \\
\text { Cohort }\end{array}$ & $\begin{array}{c}\text { Steroid } \\
\text { Cohort }\end{array}$ & \\
\hline $\mathbf{N}$ & 40 & 40 & \\
\hline Mortality (\%) & 20 & 25 & $\mathrm{p}=0.59^{* *}$ \\
\hline Operative/Non-operative & $13 / 27$ & $12 / 28$ & $\mathrm{p}=0.8^{* *}$ \\
\hline AGE (yrs) & $\begin{array}{l}63.5 \\
{[45-73]}\end{array}$ & $\begin{array}{c}59.5 \\
{[49-73]}\end{array}$ & $\mathrm{p}=0.66^{*}$ \\
\hline APACHE II & $\begin{array}{c}19 \\
{[16-27]}\end{array}$ & $\begin{array}{c}21.5 \\
{[18-26]}\end{array}$ & $\mathrm{p}=0.60^{*}$ \\
\hline APACHE II ROD (\%) & $\begin{array}{c}33.6 \\
{[22-53]}\end{array}$ & $\begin{array}{c}33.9 \\
{[21-59]}\end{array}$ & $\mathrm{p}=0.88^{*}$ \\
\hline $\begin{array}{l}\text { Patient time on SPRINT } \\
\text { (hrs) }\end{array}$ & $\begin{array}{c}129.5 \\
{[64-189]}\end{array}$ & $\begin{array}{c}116.5 \\
{[90-186]}\end{array}$ & $\mathrm{p}=0.84^{*}$ \\
\hline $\begin{array}{l}\text { Patient median blood } \\
\text { glucose (mmol/I) }\end{array}$ & $\begin{array}{c}5.8 \\
{[5.4-6.2]}\end{array}$ & $\begin{array}{c}6.0 \\
{[5.8-6.4]}\end{array}$ & $\mathrm{p}=0.13^{*}$ \\
\hline $\begin{array}{l}\text { Equivalent daily dose of } \\
\text { hydrocortisone (mg) }\end{array}$ & 0 & $\begin{array}{c}160 \\
{[80-200]}\end{array}$ & \\
\hline $\begin{array}{l}\text { Total time on Steroids } \\
\text { (hrs) }\end{array}$ & 0 & 4487 & \\
\hline $\begin{array}{l}\text { Total time on SPRINT } \\
\text { (hrs) }\end{array}$ & 7467 & 6022 & \\
\hline
\end{tabular}

$\dagger[\mathrm{IQR}]$ - Interquartile range

* p-values calculated with two-tailed Mann-Whitney U test.

** p-values calculated with chi square test.

\subsection{Model-Based Insulin Sensitivity}

This study uses the clinically validated glucose-insulin model of Lin et al (2006). The model-based insulin sensitivity has been shown to correlate well with the insulin sensitivity index (ISI) determined by the euglycaemic clamp method (Lotz et al. 2006). The glucose-insulin system model is presented in equations (1)-(3), where $S_{I}$ is the insulin sensitivity. Blood glucose level is denoted $G$, while $Q$ and $I$ represent interstitial and plasma insulin concentrations respectively. The remaining rates and constants have been previously defined in literature (Lin et al. 2006, Lotz et al. 2006).

$$
\begin{aligned}
& \dot{G}=-p_{G} G-S_{I} G \frac{Q}{1+\alpha_{G} Q}+P(t)+\frac{E G P-C N S}{V_{G}} \\
& \dot{Q}=-k Q+k I \\
& \dot{I}=-\frac{n I}{1+\alpha_{I} I}+\frac{u_{e x}}{V_{I}}
\end{aligned}
$$

Using this model and the patient data, an $S_{I}$ value is identified every hour for each patient while they are on the SPRINT protocol. If there is a gap in a patient record of longer than 5 hours (for example, when a patient is in surgery), the identification of $S_{I}$ is suspended until the record resumes. In this way, 5844 and $7149 S_{I}$ values were obtained for the steroid and control cohorts respectively.

\subsection{Analyses}

Lognormal statistics were used to provide an accurate description of the insulin sensitivity results, as typical distributions are asymmetric and skewed. For even moderately skewed distributions, the arithmetic mean is not a robust statistic, and will thus not match the defined central tendency expected. Lognormal statistics (median, multiplicative variance) are therefore a better, more robust metric. Baseline variables were compared using the twotailed Mann-Whitney $\mathrm{U}$ test or chi-square test. P-values of less than 0.05 were considered significant.

Both by-cohort and per-patient analyses of the identified insulin sensitivities were undertaken. In both cases, the data was compared using cumulative distribution functions (CDFs). CDFs show the entire shape of the distribution, which is particularly useful when they are skewed.

Examining the distribution of insulin sensitivities for each cohort provides information about the overall behaviour of a population. However, it is often also useful to look at the behaviour of per-patient or percentile patient results. For example, the theoretical median patient can shed light on how a typical patient from a population might behave even when it is difficult or impossible to actually identify a specific individual example. Similarly, more extreme percentile patients, such as at the $10^{\text {th }}$ - or $90^{\text {th }}$-percentile can show how more extreme or variable patients might behave within this cohort. 
Theoretical percentile patients are 'created' from the perpatient CDFs of the cohort at several likelihood values. At each likelihood, the desired percentile value of insulin sensitivity is determined across all individual patients and this value forms part of the theoretical percentile patient's CDF. An example is illustrated in Figure 1 at three likelihood values for the $50^{\text {th }}$-percentile patient.

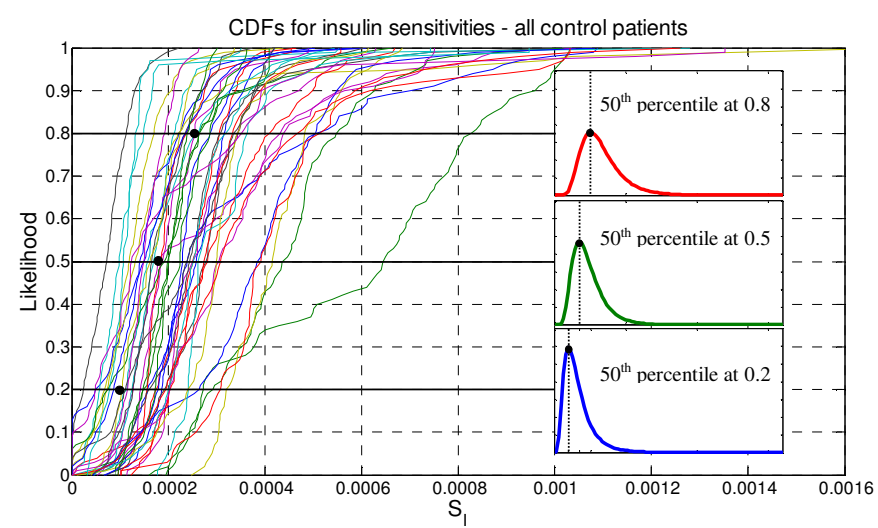

Figure 1. Per-patient analysis - creating the theoretical median patient.

Thus, the following comparisons of median $S_{I}$ are made:

- Overall cohort.

- On/off steroids.

- First/last 24hrs (or half) of treatment.

- $25^{\text {th }}, 50^{\text {th }}$ and $75^{\text {th }}$ percentile patients.

- $\Delta S_{I}$ over these patients

\section{RESULTS}

\subsection{Overall cohort analysis}

The insulin sensitivities of patients receiving corticosteroids are only slightly lower than the control patients when the cohorts are compared. The median insulin sensitivity is reduced by 9 -percent from $2.06 \times 10^{-4}$ - to $1.87 \times 10^{-4} 1 / \mathrm{mU}$.min $(\mathrm{p}<0.001)$.

Within the steroid cohort, comparing the insulin sensitivities between the periods when the patients were receiving corticosteroids and the periods when they were not also shows a reduction in sensitivity. The median insulin sensitivity is reduced by 13-percent, from $2.16 \times 10^{-4}$ 1/mU.min to $1.87 \times 10^{-4} 1 / \mathrm{mU}$.min while receiving steroids ( $\mathrm{p}<$ $0.001)$. This latter result may be more a reflection of improvement in patient condition over time than a result of the steroid treatment. Just 172 of the 1535 hours $(11 \%)$ of data during which patients in the steroid cohort were not receiving steroids were prior to treatment being commenced. The cumulative distribution functions for the insulin sensitivities of the cohorts are shown in Figure 2.

Examining the insulin sensitivities for just the first and last 24 hours (or half of stay if less than 48 hours) of patients receiving steroids shows a clear increase over the course of the treatment. The median insulin sensitivity increases by $32-$ percent over time $(\mathrm{p}<0.001)$. This improvement is mirrored, to a lesser extent, in the control cohort, where a 12-percent increase in the median is observed $(\mathrm{p}<0.001)$. These results are shown in Figure 3 where the controls are higher valued, as in Figure 2.

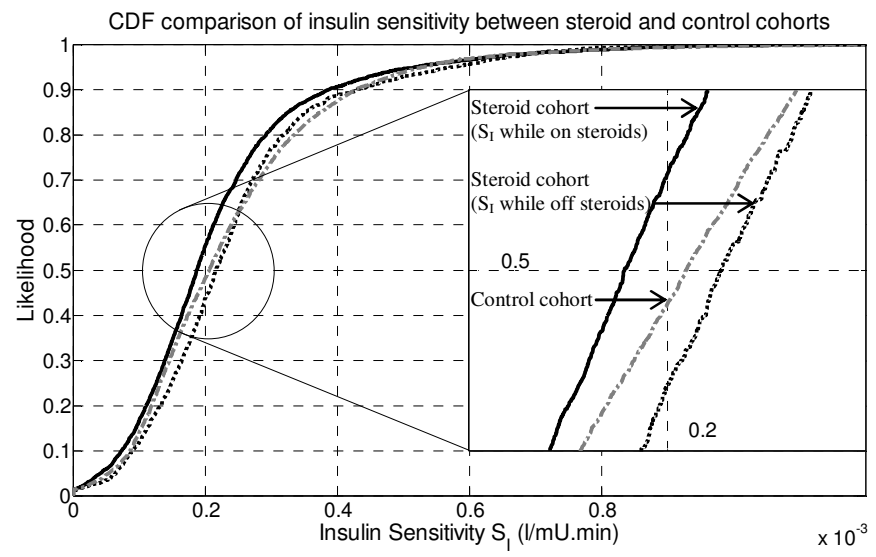

Figure 2. CDFs of insulin sensitivities for the study cohorts.

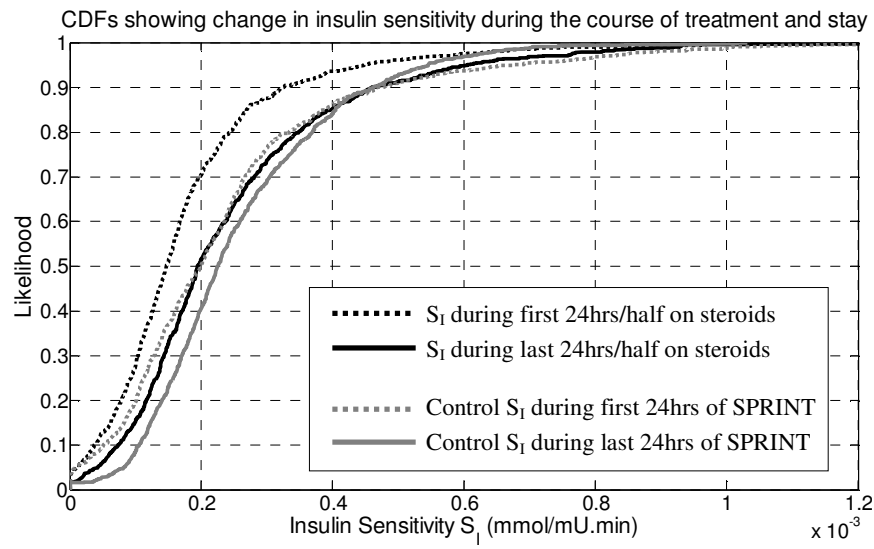

Figure 3. Evolution of insulin sensitivity during the course of treatment and stay for each cohort.

\subsection{Per-patient analysis}

A per-patient analysis of the data also shows a reduction in insulin sensitivity for those patients receiving corticosteroids. Figure 4 shows the cumulative distribution functions for the $25^{\text {th }}$-, $50^{\text {th }}$ - and $75^{\text {th }}$-percentile patients from both cohorts. There is a clear difference at all likelihood values.

It can be seen that lower percentile patients have less of a difference than higher percentile patients. The $25^{\text {th }}$-percentile patient receiving corticosteroids has median insulin sensitivity reduced by $2.36 \times 10^{-5} 1 / \mathrm{mU} \cdot \min (15 \%)(\mathrm{p}=0.007)$ when compared with the $25^{\text {th }}$-percentile patient from the control cohort. The median and $75^{\text {th }}$-percentile patients have reductions of $3.99 \times 10^{-5} \mathrm{l} / \mathrm{mU} \cdot \mathrm{min}(18 \%)(\mathrm{p}=0.002)$ and $5.01 \times 10^{-5} 1 / \mathrm{mU} \cdot \min (18 \%)(\mathrm{p}=0.002)$, respectively.

This trend can be seen more clearly in Figure 5, where the percent change in median insulin sensitivity is plotted against the patient percentile and a linear, least squares fit is shown. 
Data for patients more extreme than the $10^{\text {th }}$ and $80^{\text {th }}$ percentile is omitted, as the number of points making up the percentile patients is small and skewed by outlying values.

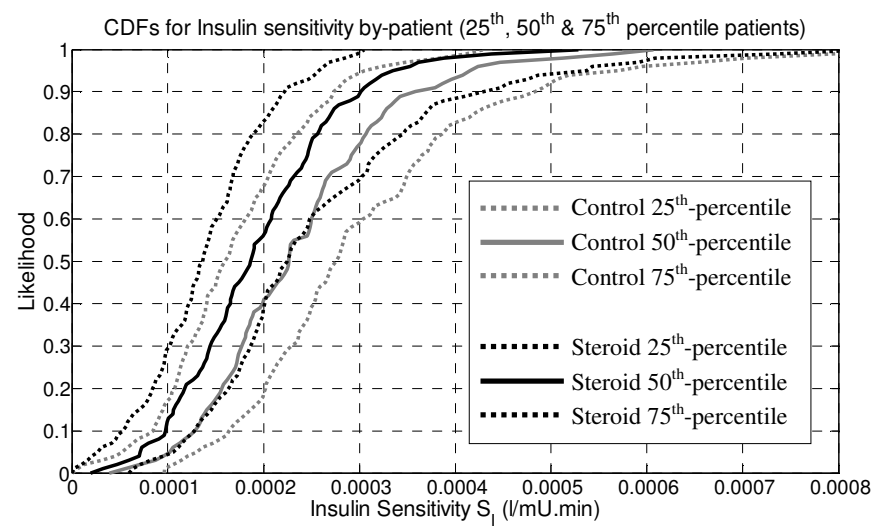

Figure 4. CDFs of insulin sensitivity for the 25th-, 50th- \& 75th-percentile patients from the study cohorts

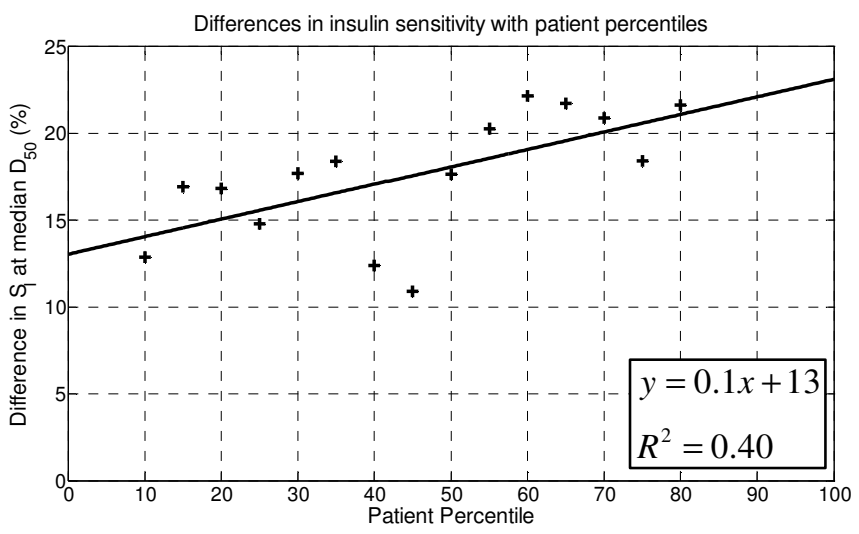

Figure 5. Differences in insulin sensitivity across theoretical percentile patients.

Figures 6 and 7 plot the distribution of insulin sensitivities for each of the 40 patients in the control and steroid cohorts. These plots clearly show that the outlying patients with higher insulin sensitivities tend to be highly variable and will therefore influence the more extreme theoretical percentile patients.

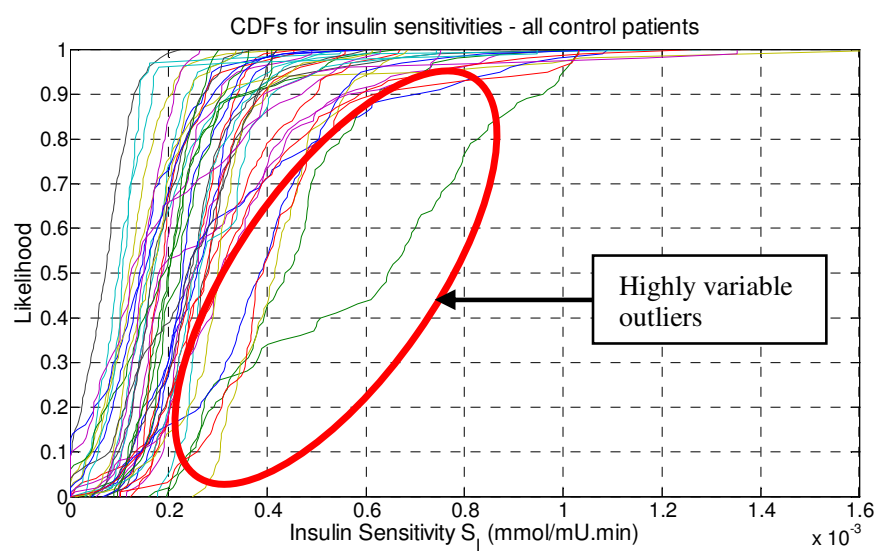

Figure 6. CDFs of insulin sensitivities for all control patients.
The majority of patients however show a tight central tendency with a very narrow interquartile range at the 0.5 likelihood $\left(8.88 \times 10^{-5} \mathrm{l} / \mathrm{mU}\right.$.min for the steroid cohort and $1.16 \times 10^{-4} 1 / \mathrm{mU}$.min for the control cohort).

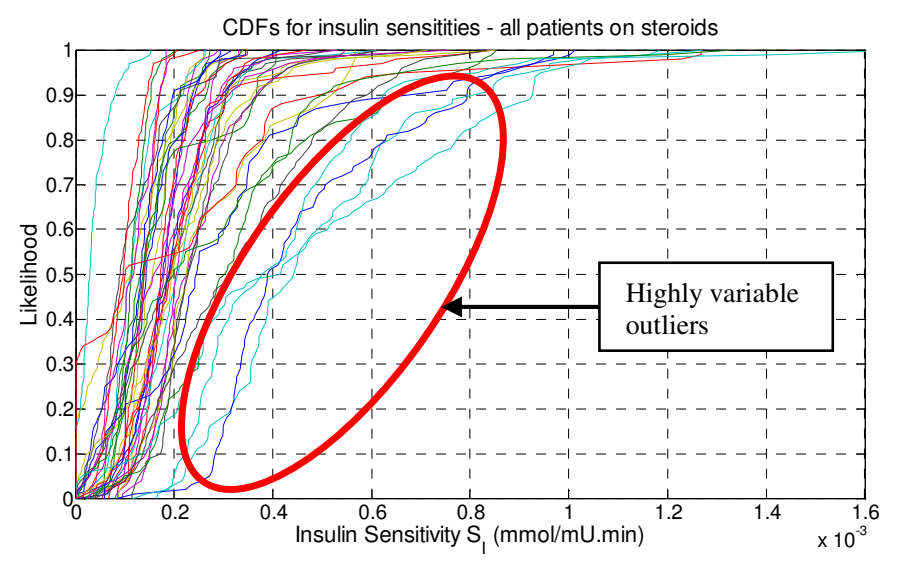

Figure 7. CDFs of insulin sensitivities for all steroid patients.

\section{DISCUSSION}

Corticosteroids cause insulin resistance in healthy people. The aim of this research was to determine whether this change is also true of critically ill patients who are already significantly insulin resistant. The results indicate that there is reduction in insulin sensitivity in critically ill patients associated with the use of corticosteroids. However, this reduction is significantly less than that seen in healthy people.

In this study, a 9-percent reduction in median insulin sensitivity was seen between patients receiving corticosteroids while on steroids and the control cohort. The dosage and particular drug received by patients varied between individuals and over the course of treatment. However, over the entire cohort, the median per-patient daily dose was equivalent to 160 [80-200] mg/d of hydrocortisone.

In contrast, the 30-62-percent reduction in insulin sensitivity reported in healthy subjects (Binnert et al. 2004, Nicod et al. 2003, Pagano et al. 1983, Perry et al. 2003) was obtained with significantly lower steroid doses. Subjects in these studies were either administered with $2 \mathrm{mg} / \mathrm{d}$ of dexamethasone, equivalent to $50 \mathrm{mg} / \mathrm{d}$ of hydrocortisone (Binnert et al. 2004, Nicod et al. 2003, Perry et al. 2003) or $15 \mathrm{mg} / \mathrm{d}$ of prednisone, equivalent to $60 \mathrm{mg} / \mathrm{d}$ of hydrocortisone (Pagano et al. 1983). Larsson and Ahren (1996) reported a 45-percent reduction with $6 \mathrm{mg} / \mathrm{d}$ dexamethasone (equivalent to $150 \mathrm{mg} / \mathrm{d}$ ) hydrocortisone).

Thus, healthy subjects received 62- 68-percent lower doses. The differences therefore cannot be attributed to dosing. Hence, there is a clear reduction in the suppression of $S_{I}$ in the critically ill. However, it is important to note that critically ill patients have $S_{I}$ values 8-60-times lower than healthy subjects (Lin et al. 2006, Lotz et al. 2006).

Within the steroid cohort, there is a 13-percent reduction in median insulin sensitivity between the periods during which 
patients were receiving corticosteroids and the periods when they were not. However, this result is probably more a reflection of improvement in patient condition than the effects of steroids. In particular, only 172 hours of data was prior to treatment with corticosteroids, compared with 1363 hours after treatment had ceased. Therefore, over 89-percent of insulin sensitivity values for the sub-cohort not receiving steroids came from the latter part of patients' stays where improved condition would be expected in general.

Figure 3 clearly shows that the insulin sensitivity values for patients increase over the course of their steroid treatment, so it seems that most, if not all, of the difference in insulin sensitivity within the steroid cohort can be attributed to improvement in patient condition over time, rather than the effects of corticosteroids. This increase over time matches clinical expectations (Chase et al. 2008).

Comparing the insulin sensitivities on a per-patient basis confirms the results seen between cohorts. The reduction in median insulin sensitivity observed for theoretical percentile patients (11- 22-percent) is more than that seen over the cohort. However, it is still much less than seen in healthy people.

Interestingly, looking between the $10^{\text {th }}$ - and $80^{\text {th }}$-percentiles where the data is dense enough to provide a reliable distribution, there appears to be a linear relationship where the lower percentile patients have less of a reduction in insulin sensitivity and the higher percentile patients more. This trend indicates that patients with higher effective insulin sensitivities tend to get more suppression, while on corticosteroids. This relationship suggests that the mechanisms by which corticosteroids affect insulin sensitivity are saturable and may already be at or near saturation in critically ill patients, given their much lower $S_{I}$ compared with healthy individuals.

Although much research has been conducted on the effects of corticosteroids on insulin sensitivity, very little is known about the mechanisms that cause them. Corticosteroids reduce insulin sensitivity directly, as well as disrupting glucose metabolism at the liver, pancreas and peripheral tissues.

A number of studies have indicated that decreased cellular glucose uptake is at least partly responsible (Pagano et al. 1983, Paquot et al. 1995, Tappy et al. 1994). However, it is unclear whether this result is due to reduced insulin binding at the receptor or disrupted glucose transport. Tappy et al (1994) showed that the decreased uptake cannot be attributed to reduced peripheral blood flow caused by the inhibition of insulin mediated vasodilatory action. Impaired intracellular glucose oxidation has also been shown to have a role in corticosteroid induced insulin resistance (Paquot et al. 1995, Tappy et al. 1994).

Delaunay et al (1997) and Lambillotte et al (1997) show that corticosteroids suppress insulin secretion through a direct action on the pancreatic $\beta$-cells. However, the results from Binnert et al (2004), Besse et al (2005) and Nicod et al (2003) show a clear increase in glucose-induced insulin secretion after administration of dexamethasone. Perhaps there are competing pathways with the net affect depending upon specific physiological conditions.

Endogenous glucose production is enhanced by corticosteroids. Increases of 28- 83-percent have been reported (Besse et al. 2005, Binnert et al. 2004, Nicod et al. 2003, Pagano et al. 1983) Some of the variation is explained by the levels of suppression achieved by hyperinsulinaemia during the clamp procedure. Catecholamines also stimulate EGP (Barth et al. 2007, Rizza et al. 1980) and this may occur through a common mechanism.

Corticosteroids are known to interact with the sympathoadrenal system, enhancing the synthesis and actions of catecholamines (Paquot et al. 1995, Taylor \& Hancox 2000). The study by Paquot et al (1995) suggests that corticosteroids and sympathomimetic agents may impair glucose metabolism through common actions. Corticosteroids may therefore affect glucose metabolism and insulin sensitivity through a signalling pathway involving or intersecting with catecholamines and the sympathetic nervous system.

The insulin sensitivity parameter in the model used for this research captures the relative net effect of altered EGP, endogenous insulin secretion $\left(\mathrm{U}_{\text {end }}\right)$, and peripheral and hepatic insulin mediated glucose uptake. Therefore, increased EGP, reduced insulin secretion or reduced insulin mediated glucose uptake result in a decrease in $S_{I}$. Hence, corticosteroid mediated changes to glucose metabolism, in addition to the direct effect on insulin sensitivity, cause a relative reduction in the model-based $S_{I}$. While taking into account net relative changes in these other metabolic parameters, the model-based $S_{I}$ still correlates very well ( $\mathrm{r}=$ 0.97) with clamp based insulin sensitivity, ISI (Lotz et al. 2006), providing support for this overall analysis.

Critically ill patients have elevated levels of circulating catecholamines and cortisol due to their stress response (Bessey \& Lowe 1993, Mizock 2001). Any increase or enhancement of their action may have little effect due to saturation. The increased hepatic glucose production associated with corticosteroids may also be blunted as it is already significantly enhanced due to the patients' condition.

Healthy individuals, in contrast, typically have low levels of catecholamines and cortisol. They would therefore show much more significant reductions in $S_{I}$ and increases in EGP with the administration of corticosteroids. This difference could explain the limited suppression of $S_{I}$ in critically ill patients receiving corticosteroids seen in this research, compared with results reported for healthy subjects.

\section{CONCLUSIONS}

This research has shown that corticosteroids cause a much lower reduction in the insulin sensitivity of critically ill patients. A 9-percent reduction in median insulin sensitivity was seen between patients receiving corticosteroids and the control cohort. In addition, patients with higher effective insulin sensitivities tend to get more suppression. This result is in contrast to healthy individuals, where corticosteroids 
result in a 30- 62-percent reduction in $S_{I}$. These results suggest that the mechanisms and pathways by which corticosteroids affect insulin sensitivity and glucose metabolism are saturable and are already at or near saturation in critically ill patients. Use of corticosteroid treatment is therefore likely to have a much less significant impact on glycaemic control in the ICU setting than might be anticipated. However, this affect is also variable among patients, creating greater variability in the control problem.

\section{REFERENCES}

Barth, E., Albuszies, G., Baumgart, K., Matejovic, M., Wachter, U., Vogt, J., Radermacher, P.and Calzia, E. (2007) Glucose metabolism and catecholamines. Crit Care Med, 35(9 Suppl), S508-18.

Besse, C., Nicod, N.and Tappy, L. (2005) Changes in insulin secretion and glucose metabolism induced by dexamethasone in lean and obese females. Obes Res, 13(2), 306-11.

Bessey, P. Q.and Lowe, K. A. (1993) Early hormonal changes affect the catabolic response to trauma. Ann Surg, 218(4), 476-89; discussion 489-91.

Binnert, C., Ruchat, S., Nicod, N.and Tappy, L. (2004) Dexamethasone-induced insulin resistance shows no gender difference in healthy humans. Diabetes Metab, 30(4), 321-6.

Bistrian, B. R. (2001) Hyperglycemia and infection: which is the chicken and which is the egg? JPEN J Parenter Enteral Nutr, 25(4), 180-181.

Capes, S. E., Hunt, D., Malmberg, K.and Gerstein, H. C. (2000) Stress hyperglycaemia and increased risk of death after myocardial infarction in patients with and without diabetes: a systematic overview. Lancet, 355(9206), 773-778.

Chase, J. G., Shaw, G., Le Compte, A., Lonergan, T., Willacy, M., Wong, X.-W., Lin, J., Lotz, T., Lee, D.and Hann, C. (2008) Implementation and evaluation of the SPRINT protocol for tight glycaemic control in critically ill patients: a clinical practice change. Crit Care, 12(2), R49.

Delaunay, F., Khan, A., Cintra, A., Davani, B., Ling, Z. C., Andersson, A., Ostenson, C. G., Gustafsson, J., Efendic, S.and Okret, S. (1997) Pancreatic beta cells are important targets for the diabetogenic effects of glucocorticoids. J Clin Invest, 100(8), 2094-8.

Derendorf, H., Hochhaus, G., Mollmann, H., Barth, J., Krieg, M., Tunn, S.and Mollmann, C. (1993) Receptorbased pharmacokinetic-pharmacodynamic analysis of corticosteroids. J Clin Pharmacol, 33(2), 115-23.

Krinsley, J. S. (2003) Decreased mortality of critically ill patients with the use of an intensive glycemic management protocol. Crit Care Med, 31, A19.

Lambillotte, C., Gilon, P.and Henquin, J. C. (1997) Direct glucocorticoid inhibition of insulin secretion. An in vitro study of dexamethasone effects in mouse islets. J Clin Invest, 99(3), 414-23.

Larsson, H.and Ahren, B. (1996) Short-term dexamethasone treatment increases plasma leptin independently of changes in insulin sensitivity in healthy women. $J$ Clin Endocrinol Metab, 81(12), 4428-32.
Lin, J., Lee, D., Chase, J., Hann, C., Lotz, T.and Wong, X. (2006) Stochastic Modelling of Insulin Sensitivity Variability in Critical Care. Biomed Sig Proc \& Control, 1, 229-242.

Lotz, T. F., Chase, J. G., McAuley, K. A., Lee, D. S., Lin, J., Hann, C. E.and Mann, J. I. (2006) Transient and steady-state euglycemic clamp validation of a model for glycemic control and insulin sensitivity testing. Diabetes Technol Ther, 8(3), 338-46.

McCowen, K. C., Malhotra, A.and Bistrian, B. R. (2001) Stress-induced hyperglycemia. Crit Care Clin, 17(1), 107-124.

Melby, J. C. (1977) Clinical pharmacology of systemic corticosteroids. Annu Rev Pharmacol Toxicol, 17, 511-27.

Mizock, B. A. (2001) Alterations in fuel metabolism in critical illness: hyperglycaemia. Best Pract Res Clin Endocrinol Metab, 15(4), 533-51.

Nicod, N., Giusti, V., Besse, C.and Tappy, L. (2003) Metabolic adaptations to dexamethasone-induced insulin resistance in healthy volunteers. Obes Res, 11(5), 625-31.

Pagano, G., Cavallo-Perin, P., Cassader, M., Bruno, A., Ozzello, A., Masciola, P., Dall'omo, A. M.and Imbimbo, B. (1983) An in vivo and in vitro study of the mechanism of prednisone-induced insulin resistance in healthy subjects. J Clin Invest, 72(5), 1814-20.

Paquot, N., Schneiter, P., Jequier, E.and Tappy, L. (1995) Effects of glucocorticoids and sympathomimetic agents on basal and insulin-stimulated glucose metabolism. Clin Physiol, 15(3), 231-40.

Perry, C. G., Spiers, A., Cleland, S. J., Lowe, G. D., Petrie, J. R.and Connell, J. M. (2003) Glucocorticoids and insulin sensitivity: dissociation of insulin's metabolic and vascular actions. J Clin Endocrinol Metab, 88(12), 6008-14.

Rizza, R. A., Cryer, P. E., Haymond, M. W.and Gerich, J. E. (1980) Adrenergic mechanisms for the effects of epinephrine on glucose production and clearance in man. J Clin Invest, 65(3), 682-9.

Tappy, L., Randin, D., Vollenweider, P., Vollenweider, L., Paquot, N., Scherrer, U., Schneiter, P., Nicod, P.and Jequier, E. (1994) Mechanisms of dexamethasoneinduced insulin resistance in healthy humans. J Clin Endocrinol Metab, 79(4), 1063-9.

Taylor, D. R.and Hancox, R. J. (2000) Interactions between corticosteroids and beta agonists. Thorax, 55(7), 595-602.

Van den Berghe, G., Wilmer, A., Hermans, G., Meersseman, W., Wouters, P. J., Milants, I., Van Wijngaerden, E., Bobbaers, H.and Bouillon, R. (2006) Intensive insulin therapy in the medical ICU. $N$ Engl J Med, 354(5), 449-61.

Van den Berghe, G., Wouters, P., Weekers, F., Verwaest, C., Bruyninckx, F., Schetz, M., Vlasselaers, D., Ferdinande, P., Lauwers, P.and Bouillon, R. (2001) Intensive insulin therapy in the critically ill patients. N Engl J Med, 345(19), 1359-1367. 